\title{
MODELLING AND PERFORMANCE EVALUATION OF FLEXIBLE MANUFACTURING SYSTEMS USING DETERMINISTIC AND STOCHASTIC TIMED PETRI NETS*
}

\author{
T.K. Behera, B.S. Mishra, L.M. Patnaik
}

C. Girault

\begin{abstract}
This paper describes a Petri net-based methodology that has been developed for the performance analysis of Real-Time Systems (RTS). A Flexible Manufacturing System (FMS), which is a case of a real-time system, has been used as a vehicle for our discussion. We use here Deterministic and Stochastic methods for modelling and analysing the performance of this Real-Time systems. Since the operational times of an FMS, such as machine times and part transfer times, are deterministic, we use Deterministic Timed Petri Nets (DTPNs) for obtaining the performance of the system modelled; whereas for analysing tool breakage and machine failure we use Generalised Stochastic Petri Nets, because such failures are random in nature. We use a software package developed inhouse for the deterministic timed analysis, whereas we use the GreatSPN1.3 for stochastic analysis.
\end{abstract}

Keywords: Deterministic Timed Petri Nets, Generalised Stochastic Petri Nets, Flexible Manufacturing Systems, Semi-Markov Chain, Performance Analysis.

\section{INTRODUCTION}

Petri Nets are becoming increasingly popular as a powerful modelling tool for the modelling and analysis of systems that exhibit both concurrent and asynchronous activities. Petri nets are well-suited for specifying and modelling dynamic properties of real-time systems. Some of the important characteristics of Petri nets that make them especially suitable for specification, modelling, and verification of systems are the following.
- Petri nets support explicit representation of causal dependencies.

- Petri net models allow description of systems at different levels of abstractions, which is valuable for the modelling of hierarchical systems.

- Petri nets are graphical formalisms, therefore the models of a system behavior are easily understood.

Although basic Petri net[1] models can be used to model the flow of control, concurrence, and synchronization among events, Petri nets possess no means of expressing time that can be used to study system performance. To evaluate system performance, researchers $[2,3,4]$ have introduced the notion of time in Petri nets in the form of delays associated with transitions or places. We refer to these models as Timed Petri Nets(TPNs). The firing delay of a transition can be specified either deterministically or stochastically. Deterministic Timed Petri Nets (DTPNs), are a class of Timed Petri Nets, in which for some transitions the delay is specified by a constant or a finite time interval and others are immediate transitions with zero or no time intervals. The selection among possibly conflicting enabled immediate transitions is made through firing probabilities. Generalized Stochastic Petri Nets (GSPNs)[5] are basically a place/transition net with priorities and inhibitor arcs with an associated set of rate and marking parameters. Each transition has an associated function of rate parameter. Places are divided into two subsets: places that have fixed associated initial marking, and places that have a function of the marking parameters as initial marking. Transitions that fire in zero time and with priority over stochastic transitions, are called immediate transitions. In GSPNs the transitions that are associated with an exponentially distributed random variable for the time 
to fire the transition are said to be stochastic transitions.

In this paper, we have focussed our attention on performance modelling of a Flexible Manufacturing Systern using both deterministic and stochastic time Petri nets. Since with known machine times and part transfer times, the throughput rate, and the average machine utilization of the system can be known in advance; we use a Deterministic Timed Petri Net Simulator(DTPNS) for solving such a problem. But some events like occurrence of machine failures and repairing of machine tools may occur randomly at any time during the process of operation of the system; so the use of DTPNS for studying such systems doesn't give a realistic result. We use here the Generalized Stochastic Petri Net(GSPN) for evaluating the performance of such a system with random failures and consequent repairs.

A software package (developed in the Microprocessor Applications Laboratory, Indian Institute of Science, Bangalore, India) that generates the state space of a DTPN model has been used to construct the reachability graph and to get the steady state probability distributions. This package has been used for the performance evaluation study of the deterministic case. We use GreatSPN 1.3[10] package for studying the performance of the system with stochastic behavior. The performance paramelers of interest in our study are throughput rate and machine utilization, with different scheduling policies of jobs and probabilities of machine breakage. The objective of our study is to arrive at a scheduling scheme which ensures a balanced utilization of various resources of FMSs, such as machines, tools and fixture in order to guarantee maximum throughput and maximum machine utilization.

This paper is organized as follows. In the next section, we give a description on DTPNs and GSPNs. Section III describes the modelling of a simple Flexible Manufacturing System using DTPN arid GSPN as modelling tools.In section IV, we show our results obtained from these models. Section V concludes this paper.

\section{TIMED PETRI NETS(TPNs)}

One major extension over the past in PN modelling is inclusion of time into the net. Time is included in a PN model; such a net is referred as Timed Petri Nets (TPNs). Timed Petri nets have been used for the performance evaluation of concurrent systems[7] and, in particular, of communication protocols[9] and multiprocessor computer systems[5]. Time is associated with the transitions and when enabled or fired, the firing delay of the transitions can be either deterministic or stochastic in nature. Due to this nature of transition firing, Timed Petri Nets are grouped into Deterministic Timed Petri Nets(DTPNs) and Generalized Stochastic Petri Nets(GSPNs). Transitions called immediate transitions that takes zero time or no time to fire are another extension to the nature of Timed Petri Net transitions. In the following subsections, we describe features related to DTPNs and GSPNs.

\subsection{Deterministic Timed Petri Nets (DTPNs)}

In this section, we discuss some properties of D'I'l'Ns. Deterministic Timed Petri nets are a class of timed Petri nets where a constant delay is augmented to the transitions of the net. Then a DTPN is defined as, $N=(P, T, E, W, H, 6)$ where,

$P$ : Finite nonempty set of places

$T$ : Finite nonempty set of transitions

$E$ : A set of directed arcs that connects places to transitions and vice versa

$$
E \subseteq P \times T \cup T \times P
$$

such that,

$$
\begin{array}{r}
\forall(t \in T) \exists\left(p_{i}, p_{j} \in P\right)\left(p_{i}, t\right) \in E \wedge\left(t, p_{j}\right) \in E \\
W: E N^{+} \text {(weight of the arcs) }
\end{array}
$$

$H$ : Finite set of inhibitor arcs such that,

$$
\text { 6:T } \begin{array}{r}
H \subseteq P \times \\
N+(\text { duration of firing) }
\end{array}
$$

A place is an input (or an output) place of a transition $t$ iff there exists an $\operatorname{arc}$ from $(p$ to $t$ ) or (from $t$ top) respectively in the set $E$. The sets of all input and output places of a transition $t$ are denoted by $\operatorname{Inp}(t)$ and Out $(t)$, respectively. Similarly, the set of all input and output transitions of a place is denoted by $\operatorname{Inp}(p)$ and $\operatorname{Out}(p)$ respectively. A place is denoted by $\operatorname{Inh}(t)$ if there exists an inhibitor arc from $p$ to $t$.

2.1.1 Marking - A marking of a Timed Petri net is a pair $(\mathrm{M}, F)$ where,

$M: P-N^{+}$(number of tokens in a place)

$F: \psi-N^{+}$(remaining firing time)

where;

$\psi$ is the set of transitions which have initiated and have not yet completed the firing process.

We call $\mathrm{M}$ the marking of places and $F$ the remaining firing time of transitions. In basic Petri nets, the markings of places are only defined. The marking $M$ is the number of tokens present in a place at any instant of time. It may appear that the marking $M$ is sufficient to define the state of the system. Since the tokens are distributed in places as well as in those transitions waiting for the completion of firings, the remaining firing 
time information of the transitions waiting to complete the firing process is also necessary. Remaining firing time $(F)$ represents the time a transition needs more to complete the firing that is in progress.

2.1.2 Firing rules - $\mathbf{A}$ transition $\boldsymbol{t}$ is enabled iff, $\forall\left(p_{i} \in \operatorname{Inp}(t)\right) M\left(p_{i}\right) \geq W\left(p_{i}, t\right)$ A $\forall p_{i} \in$ $\operatorname{Inh}(t) M\left(p_{i}\right)=0$

All enabled transitions initiate firing simultaneously removing the required number of tokens from the input places as soon as they become enabled. The new markings after initiating the firing of a transition $t$ are given by,

$M\left(p_{i}\right)=M\left(p_{i}\right)+W\left(p_{i}, t\right)$ if $p i \in \operatorname{Inp}(t)$

$=M\left(p_{i}\right.$ if $p_{i} \notin \operatorname{Inp}(t)$

$F(t)=\delta(t)$

All the transitions whose $F$ values become zero, complete firing simultaneously by adding the required number of tokens to the output places. The new markings after transition $t$ completes firing are given by,

$M\left(p_{i}\right)=M\left(p_{i}\right)+W\left(p_{i}, t\right)$ if $p_{i} \in$ Out $(t)$

$=M\left(p_{i}\right)$ if $p_{i} \notin O u t(t)$

$F(t)=$ undefined in case of disabled transitions

A situation where the firing of one transition disables some other transition is called a conflicting case. In a conflicting case, the conflicting transitions are fired with the probabilities associated with them.

\subsection{Generalized Stochastic Petri Nets(GSPNs)}

Generalized Stochastic Petri Nets(GSPNs) [5] are a particular class of Timed Petri Nets(TPNs) introduced in order to improve the effectiveness of SPNs. Then a GSPN is defined as, $N=(\mathrm{P}, T, E, W, H, \delta)$ where,

$\mathrm{P}$ : Finite nonempty set of places

$T$ : Finite nonempty set of transitions. The set $T$ is partitioned into two separate sets $T_{i}$ and $T_{e} . T_{i}$ is the set of immediate transitions and $T_{e}$ is the set of transitions called exponential transitions.

$E$ : A set of directed arcs that connects places to transitions and vice versa

such that,

$$
E \subseteq P \times T \cup T \times P
$$

$$
\forall(t \in T) \exists\left(p_{i}, p_{j} \in P\right)\left(p_{i}, t\right) \in E \wedge\left(t, p_{j}\right) \in E
$$

$W: E \longrightarrow N^{+}$(weight of the arcs)

$H$ : Finite set of inhibitor arcs such that,

$$
H \subseteq P \times T \text { and } E \cap H=\emptyset \text {. }
$$

$6: T_{e} \longrightarrow R^{+}$( average duration of firing)

The immediate transitions have a firing time equal to zero in all the markings. The elements of $T_{e}$, which is called exponential transitions, have an exponentially distributed firing time.

2.2.1 Markings and Firing Rules - Due to the memory less property of exponential distribution, the marking of GSPNs is defined as $\boldsymbol{M}$, where

$$
M: P=N^{+} \text {(number of tokens in a place) }
$$

The enabling condition of a GSPN is same as the DTPNS. However, since the immediate transitions have priority over timed transitions, the following firing rules are followed. 1) The enabled exponential transitions are not fired when there are enabled immediate transitions. 2) If two or more concurrent immediate transitions are enabled, all of them fire simultaneously. 3) If some of the enabled immediate transitions are conflicting, only one of them is allowed to fire at a time, according to a predefined probability distribution.

The states where immediate transitions are fired are called vanishing states whereas when the exponential transitions are fired, the corresponding states are called tangible states.

\subsection{State Space Generation}

The reachability graph of a TPN is a directed graph $\Gamma$ $=(\mu, \theta, \gamma)$ where, $\mu$ is the set of reachable states of the TPN model $\theta$ is the set of directed $\operatorname{arcs}, \theta \subseteq\left(\begin{array}{lll}\mu & \mathrm{x} & \mu\end{array}\right)$, such that $\left(\mu_{i}, \mu_{j}\right) \in \theta$ iff $\mu_{j}$ is directly reachable from $\mu_{i}$, and 7 is the labelling function which assigns the probability $\gamma_{i j}$, that a transition takes place from state $\mu_{i}$ to state $\mu_{j}$

Thus, $\gamma: \theta \longrightarrow(0,1)$; that is $\gamma\left(\mu_{i}, \mu_{j}\right)=\gamma_{i j}$.

The holding times (Sojourn times) of states are greater than or equal to zero. The states with nonzero sojourn time are called Tangible states and the states with zero sojourn times are called vanishing states. As the states change in zero time in case of vanishing states, they don't contribute to the time dependent evaluation of the system.

\subsection{Steady-State Performance Estimation}

The steady state probabilities are calculated for an irreducible semi-Markov chain obtained as the state space of the DTPN[9] or the GSPN[8]. For an irreducible, aperiodic semi-Markov chain with all states recurrent non-null, it can be shown that the probability vector $V=\left[v_{0}, v_{1}, \ldots, v_{n}\right]$ is the unique stationary probability 
row vector satisfying the following equations :

$$
v_{j} \geq 0, \quad V=V P \quad \quad \sum v_{j}=1
$$

where $\mathrm{P}$ is the one-step probability matrix,

The Equation(1) can be modified into a more convenient form as,

$$
(I-P)^{T} V_{T}=0
$$

The stationary probability vector $\mathrm{V}$ can be calculated from the above equations.

If

$\left[s_{0}, s_{1}, s_{2} \ldots, s_{n}\right]$ are the sojourn times of the states, we can compute the steady-state probability vector $\boldsymbol{\pi}=\left[\pi_{0}, \pi_{1}, \ldots, \pi_{n}\right]$ from the stationary probabilities as per the following formula :

$$
\Pi_{i}=\frac{v_{i} s_{i}}{\sum_{j=0}^{t} v_{j} s_{j}}
$$

From the steady state probability the following performance measures can be computed.

\section{Probability that a particular condition $\mathrm{C}$ holds}

$$
P R O B(C)=\sum_{\mu_{j} \in S(C)} \Pi_{j}
$$

where, $S(C)$ is the set of states in which condition $C$ holds

\section{Throughput}

$$
\Pi_{i}=\frac{\sum_{i=1}^{n} v_{i} \times \text { Prob. }\left(\text { ti completesf iring in state } \mu_{i}\right)}{\sum_{i=1}^{n} v_{i} \times s_{i}}
$$

\section{FLEXIBLE MANUFACTURING SYSTEMS}

A new type of production system called the Flexible Manufacturing System(FMS) has emerged as a highly competitive manufacturing strategy in recent years to increase the productivity of industries. An FMS basically consists of a set of machine tools interconnected by an automated palletized transportation system. Main advantages of an FMS are the ability of working with different types of parts by adapting to changes in production mix and volume, and also capability of ensuring a lower but constant production in the case of accidental or programmed machine stops. For obtaining a balanced utilization'level of the resources machines, tools and fixtures, it is necessary that different types of properly matched parts be present at the same period of time in the system.

FMSs can be modelled and analysed using DTPNs as well using GSPNs. Since machine failures, tool breakages are some of the random features that can occur at any time during the process of machine execution, we have used a GSPN to model such systems. Since features like operational times of FMSs, machining times and part transfer times are deterministic, DTPNs are found to be most reallistic tools for performance modelling of FMSs. We model here a hypothetical FMS using both DTPNs and GSPNs to study its performance.

Figure 1 shows an example of a DTPN model of a simple FMS. In this model, we have two types of parts that are to be loaded and machined before leaving the system. In this model the type-one part has two phase operation schedule, while type-two parts need only one phase machining before leaving the system. The first phase of the type-one working schedule can only be accomplished by $M 1$, while the second phase of the type-one working schedule can be performed either by M2 or M3. Thus the processing of type 1 involves the following operations. A part is loaded into the system and is transported to $\mathrm{M} 1$, then either to $\mathrm{M} 2$ or $\mathrm{M} 3$ and finally it is unloaded. Since the tooling machine M3 executes the process faster in comparison to $\mathrm{M} 2$, we have taken care of this condition in our model by using inhibitor arcs. Similarly, the type 2 part has only one phase operation where the working schedule can be performed either by M2 or M3. For this case, M2 executes its processes faster than M3 does. Pallets are special types of devices required to hold the work pieces inside the system in order to be moved and processed into the FMS. Since different pallet types are needed for different types of parts, the available number of pallets thus constraints the number of work pieces flowing through the system, and consequently affects the production mix. When a raw work piece(token) enters into the system, it is probabilistically decided which path it must follow according to the fixed production mix percentage. However, after work pieces are processed, the part loses its identity, leaves the system and is replaced by a new raw part whose type has to be decided. In this FMS model, the events of loading/unloading and transport of raw and finished parts are grouped together in a simple action.

Figure 2. shows the GSPN model of the Flexible Manufacturing System. The basic operation of the GSPN model is same as that of the DTPN model. We assume here that service times are uniformly distributed and a constant delay to each machine service is associated. We have included the modelling of failure of the machines in the GSPN model. We did not (could not !) include it in the DTPN model because of the number of states in the DTPN model becomes very large. When a machine is processing a job, it is subjected to failure (breakage). Once a machine fails, the job is put back to the queue to be processed later 


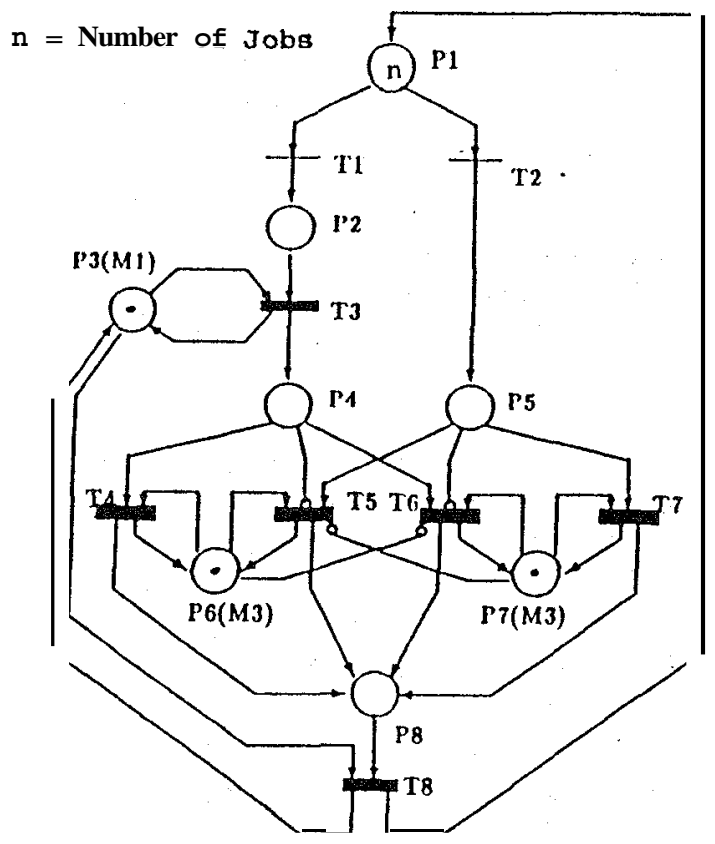

Figure 1: DTPN Model of the Flexible Manufacturing System

and the machine is subjected to repair. After the machine is repaired, it is ready to process a job. The job released due to the failure of the machine may wait for the machine to be ready again or it may be executed by another machine, if available.

The scheme of scheduling the jobs among machines in the modelled FMS can operate (though at a reduced performance) even if one of the machines M2 and M3 is down.

\section{PERFORMANCE EVALUATION OF AN FMS}

In the design and operation of an FMS that involves numerous concurrent interactions, performance modelling and evaluation play an important role. Performance evaluation involves computing performance measures such as machine utilization, production rate of parts (throughput), waiting times; using a performance model. Some of the prominent tools used in the pcrforrnance modelling arid evaluation of an FMS are Msrkov models, discrete event simulation, queuing networks etc. The DTPN model is more realistic due to the detreministic nature of the processiiig times of the jobs. Ilowever, the number of states of a DTPN is much more than the equivalent GSPN model. Due to this fact we have not incorporated the modelling of the

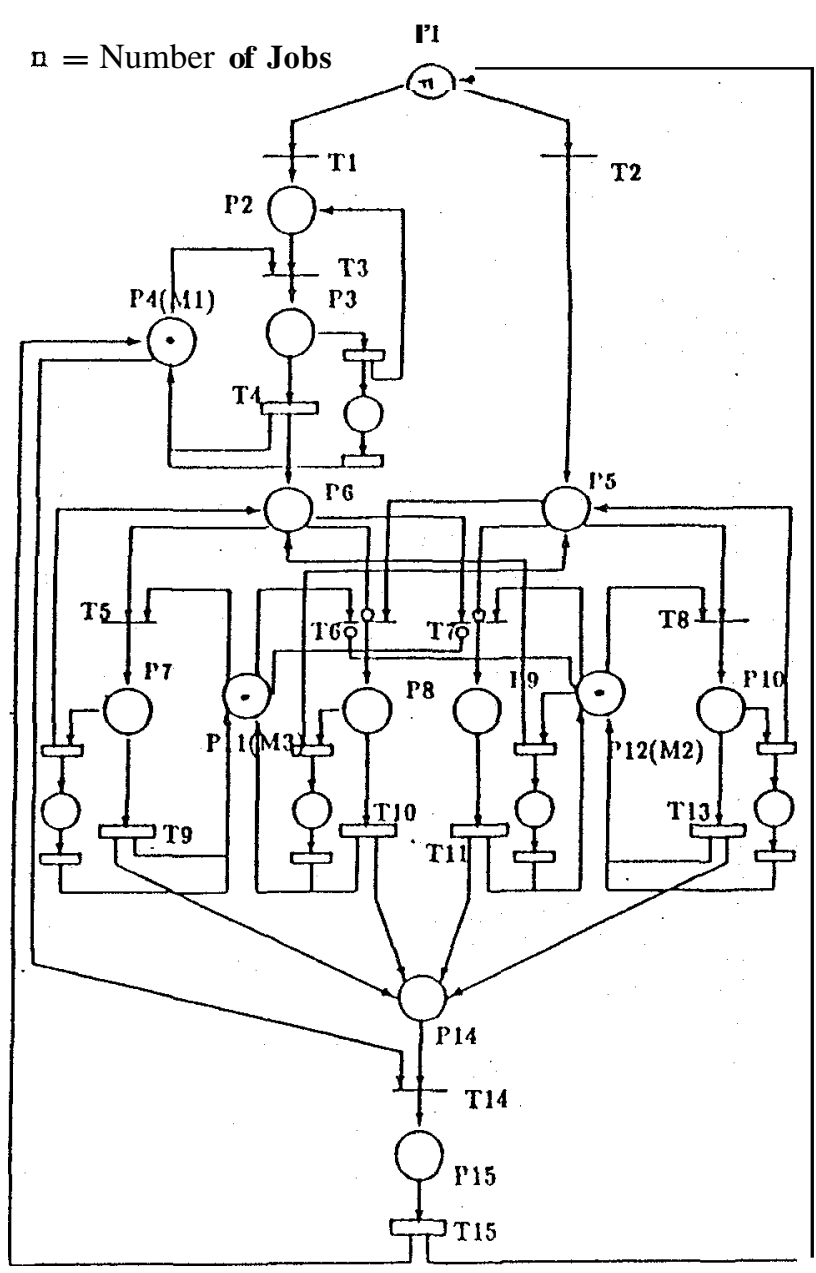

Figure 2: GSPN Model of the Flexible Manufacturing System

machine failure in the DTPN model.

The scheduling of jobs is one important feature that our model handles quite appropriately. In an FMS, parts are processed according to a job scheduling obtained with off-line basis. However, due to failure of machine tools it needs to reschedule jobs immediately on off-line or on-line basis. Rescheduling occurs automatically when there is failure with machine M2 or with machine M3. The jobs are processed cither in h12 or M3 depending on the failure of the machine. The throughput obtained from GSPN model of the FMS with machine failures is compared with that of the DTPN model of the same system. We compare throughput results of the DTPN model with the GSPN model when the systeiii is normal, i.e when there is no permanent breakdown of any macliiiie. Then we proceed to compare the throughput results of tlier GSPN model with that of the DTPN model, when machine $\mathbf{M} 2$ or h13 broaks permanently. In figure 3 , we show the different throughput results obtained. The throughput of the system is slightly more when machine M3 is down as compared to the case when machine h12 is down. ln figures $4(\mathrm{a})-(\mathrm{c})$ the idle time of machines are shown with respect to the functioning of the system, i.e. with 
and without machine breakdowns. From the figure 3, it is clear that the throughput obtained using the GSPN model is slightly less than using the DTPN model, as we have incorporated the modelling of temporary machine breakdown while it is processing a job. When a machine breaks down permanently, the throughput obtained is considerably low but the system continues to function without halt. From figure 4(a) we can see that the idle time of machine M1 is high when there is permanent breakdown of a machine (e.g. M2 or MB) .

Using the DTPNs software package developed in the Microprocessor Applications Laboratory, the evaluation of the everage machine tool idle time i.e when individual machine is free and throughput rate of the FMS has been carried out. We compare our result obtained from GSPN model using GreatSPN1.3 package of the same system.

\section{CONCLUSIONS}

In this paper we have shown the modelling power of both DTPN and GSPN for a Flexible Manufacturing system. These models have been analyzed for performance evaluation of the FMS under normal as well as abnormal cases, like permanent failure of machines. Though the deterministic time in DTPN model of the system seems to be more realistic, the analysis of the model needs lot more computational effort because of the intermediate states generated are very large compared to the equivalent GSPN model with the increase in number of jobs. It is seen that the performance measures obtained are almost equal for both DTPN and GSPN models. In the GSPN model we have included another feature, i.e. modelling the failure of a machine while it is servicing a job. Analysing this aspect using the DTPNs is difficult due to the large number of states generated.

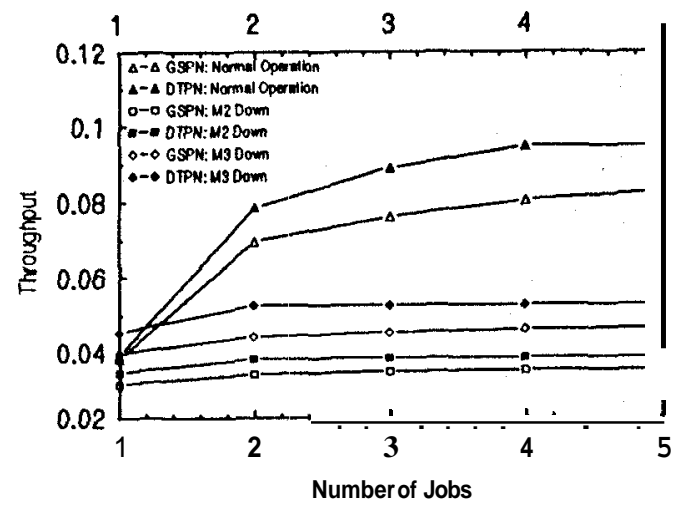

Flgure 3. Throrghpot osing DTPN and GSPN Models

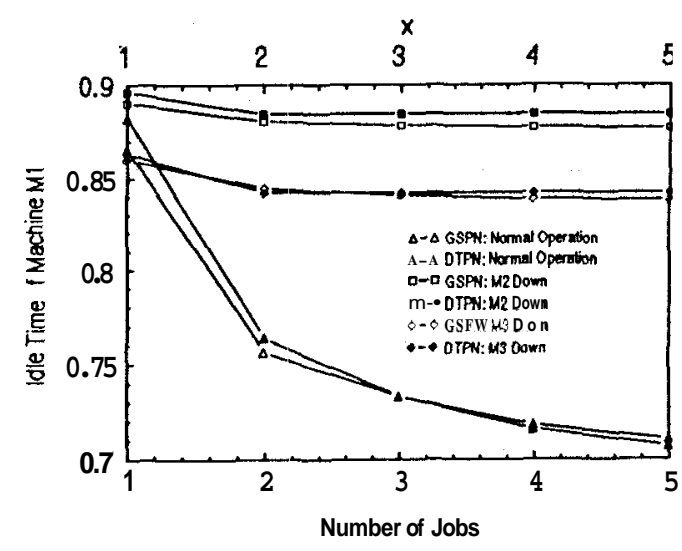

Figure 4(a) Ide Time of Machine MI using DTPN and GSPNM Models

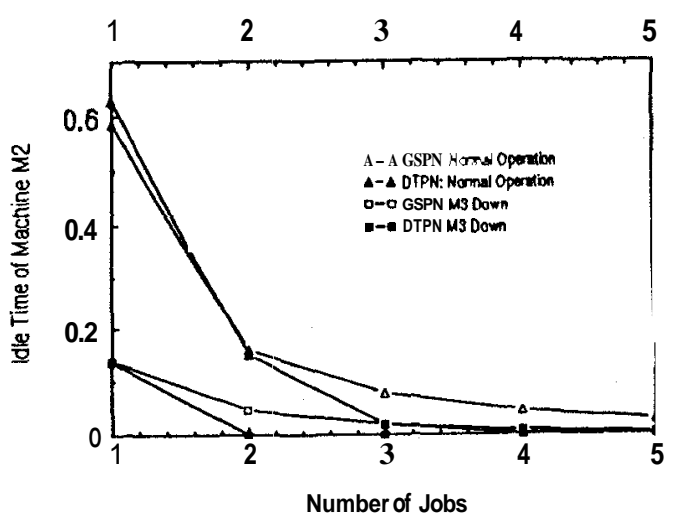

Figure 4@) Idk Time of Machine M2 usiog DTPN and GSPN Models 


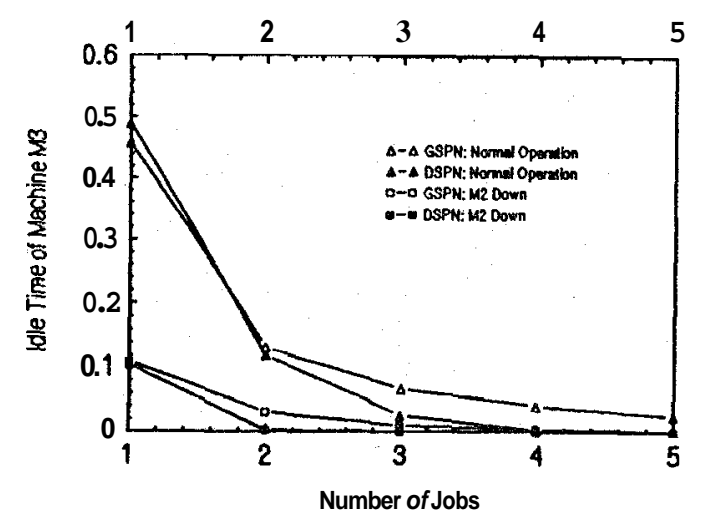

Flguro 4(c) Idk Time of Machine M3 asing DTPN and GSPN Models

[8] G.Chiola, M.A.Marasan, G.Balbo, G.Conte "Generalized Stochastic Petri Nets: A Definition at the Net Level and its Implications", IEEE Trans. on Software Engineering, Vol 19, No 2, Feb. 1993, pp. 89-102.

[9] M R El-Karaksy, A S Nouh and A Al-Obaidan "Performance Analysis of Timed Petri Net Models for Communication Protoclos: A Methodology and a Package", IEEE Communications, Vol 18, No 2, March 1990, pp. 73-82.

[10] G.Chiola, "GreatSPN USERS' Manual, Version 1.3", September 1987.

\section{References}

[1] Peterson, J.L, Petrinet Theory and Modeling of Systems, Prentice-Hall, Englewood cliffs, New Jersey, 1981.

[2] Zuberek, W.M. "Timed Petri Nets and Preliminary Performance Evaluation" Proceedings of the 7th Annual Symposium on Computer Architecture 1980, pp. 88-96.

[3] M. K. Molloy, "Performance Analysis using Stochastic Petri Nets" IEEE Transaction on Computers, Vol. 31, No. 9, pp. 913-917, 1982.

[4] Razouk, R and Phelps, V "Performance Analysis using timed Petri nets" 4th Int. Conf. on Distributed Computing Systems,May 1984 pp. 126128.

[5] M.A. Marason, G. Conte, and G. Balbo, "A Class of Generalized Stochastic Petri Nets for the Performance Evaluation of Multiprocessor Systems," ACM Transactions of Computer Systems, Vol. 2, No. 2, 1984 pp. 93-122.

[6] G.Balbo, G.Chiola, G.Franceschinis, "Generalized Stochastic Petri Nets for the Performance Evaluation of FMS", IEEE Int. Conf. on Robotics and Automation, Raleigh, USA, April,1987 pp. 10131018.

[7] C.V. Ramamoorthy and G.S.Ho, "Performance Evaluation of Asynchronous Concurrent Systems Using Petri Nets", IEEE Transactions on software Engineering. Vol. SE6, No. 5, Scpt. 1980. pp. 440449. 